\title{
Anti-malaria drug chloroquine is highly effective in treating avian influenza A H5N1 virus infection in an animal model
}

Cell Research (2013) 23:300-302. doi:10.1038/cr.2012.165; published online 4 December 2012

\section{Dear Editor,}

The recent controversial studies of man-made avian flu viruses caused a media storm, and brought new concerns to the potential of an avian influenza H5N1 virus pandemic, which has been pending since 1997 [1, 2]. Although the estimated mortality rate of avian influenza A H5N1 virus infection in humans could be as high as $60 \%$, the World Health Organization (WHO) phase of pandemic alert is currently set at 3 , due to that there has not been human-to-human or community-level transmission (http:/www.who.int/influenza/preparedness/pandemic/h5n1phase/en/index.html). However, the newly created $\mathrm{H} 5 \mathrm{~N} 1$ virus strains, which are genetically altered, are transmissible among ferrets, and thus may trigger a real pandemic that could potentially result in millions of deaths according to Science Insider [3]. While it is arguably a bit too late to debate whether regulations or mandatory reviews should be applied to these dualuse studies, in the matter of fact, these viruses that are probably among the most dangerous infectious agents known already exist. Therefore, a top priority at present is to find effective prophylactic or therapeutic agents that would help to control a pandemic of avian influenza A H5N1 viruses.

Previous reports have demonstrated that the high mortality in humans infected with avian influenza A H5N1 is partly due to acute lung injury or the resulting severe condition, acute respiratory distress syndrome (ARDS) $[4,5]$. There are few treatment choices for ARDS, aside from mechanical supporting equipment and empirical treatments. The use of cortisones is controversial.

We have recently discovered that avian influenza A H5N1 virus infection causes acute lung injury by inducing autophagic alveolar epithelial cell death [6]. Importantly, we found that autophagy inhibitors are effective in ameliorating murine acute lung injury induced by live avian influenza A H5N1 virus infections [6]. We thus hypothesize that if a drug that is currently in clinical use can act to inhibit autophagy, then such a drug might be a good candidate for treating $\mathrm{H} 5 \mathrm{~N} 1$ infections.
To test this, we have focused our efforts on chloroquine (CQ), as CQ is the only oral clinical drug that is known to be an autophagy inhibitor [7]. CQ, or N'-(7chloroquinolin-4-yl)-N,N-diethyl-pentane-1,4-diamine, was discovered in 1934 by Hans Andersag and his coworkers at Bayer Laboratories and was introduced into clinical practice in 1947 as a prophylactic treatment for malaria [8]. Currently, CQ and its hydroxyl form, HCQ, are used as anti-inflammatory agents for the treatment of rheumatoid arthritis, lupus erythematosus and amoebic hepatitis. More recently, CQ has been studied for its potential use as an enhancing agent in cancer therapies as well as novel antagonists to chemokine receptor CXCR4 in pancreatic cancer $[8,9]$.

We first tested whether CQ could inhibit cell death in the human lung carcinoma A549 cells infected with live avian influenza A H5N1 virus. The cell viability was improved both prophylactically and therapeutically in a dose-dependent manner with CQ treatments, and the efficacy of CQ was much higher than that of the potent autophagy inhibitor 3-methyladenine (3-MA) (Figure 1A). Next, we tested the potential therapeutic effect of $\mathrm{CQ}$ in a mouse model of live H5N1 infections. We found that when CQ was administered therapeutically at a dose equivalent to that for human clinical use, the survival rate of $\mathrm{H} 5 \mathrm{~N} 1$ virus-infected mice was improved dramatically (from $0 \%$ to $70 \%$ at day 8 post infection), and the body weight changes also showed a trend of improvement, but prophylactic application of CQ showed no protective effect (Figure 1B and 1C). We analyzed the lung histopathology in these mice and found fewer infiltrating leukocytes in the CQ therapeutic group, but not in the CQ prophylactic group (Figure 1D). Lung edema, as determined by the increased wet/dry weight ratio of lung tissue, was also significantly reduced by therapeutic CQ treatment, but not by prophylactic CQ treatment (Figure $1 \mathrm{E})$. Taken together, our results demonstrate that CQ, a known autophagy inhibitor that is in clinical use, could efficiently ameliorate acute lung injury and dramatically improve the survival rate in mice infected with live avian influenza A H5N1 virus. 
A

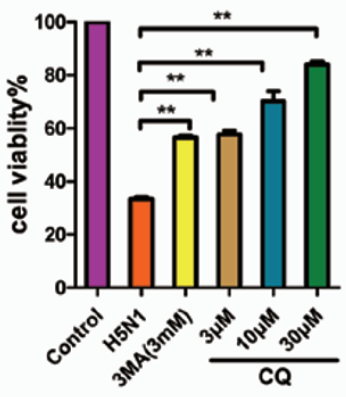

C

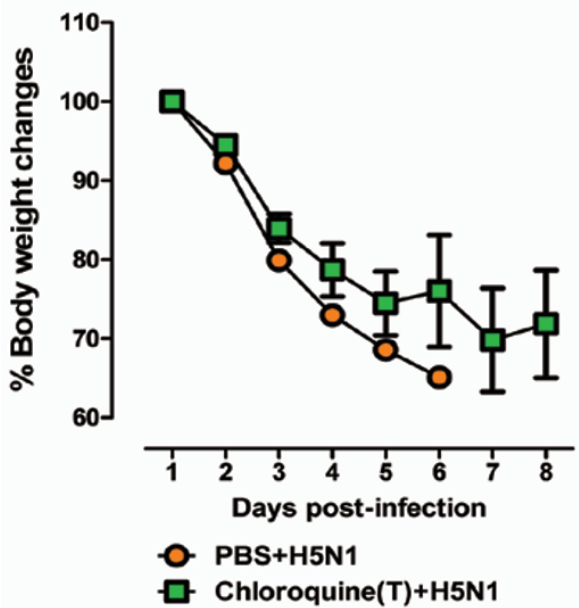

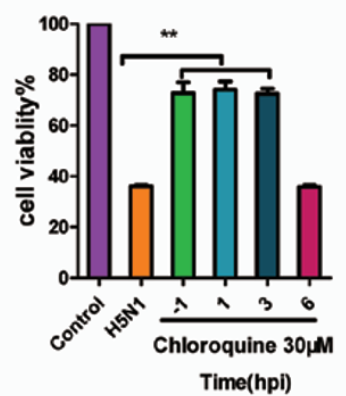
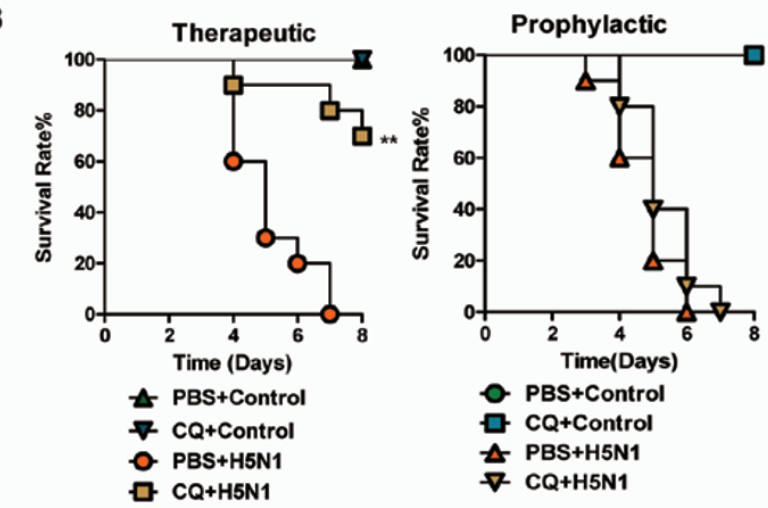

D
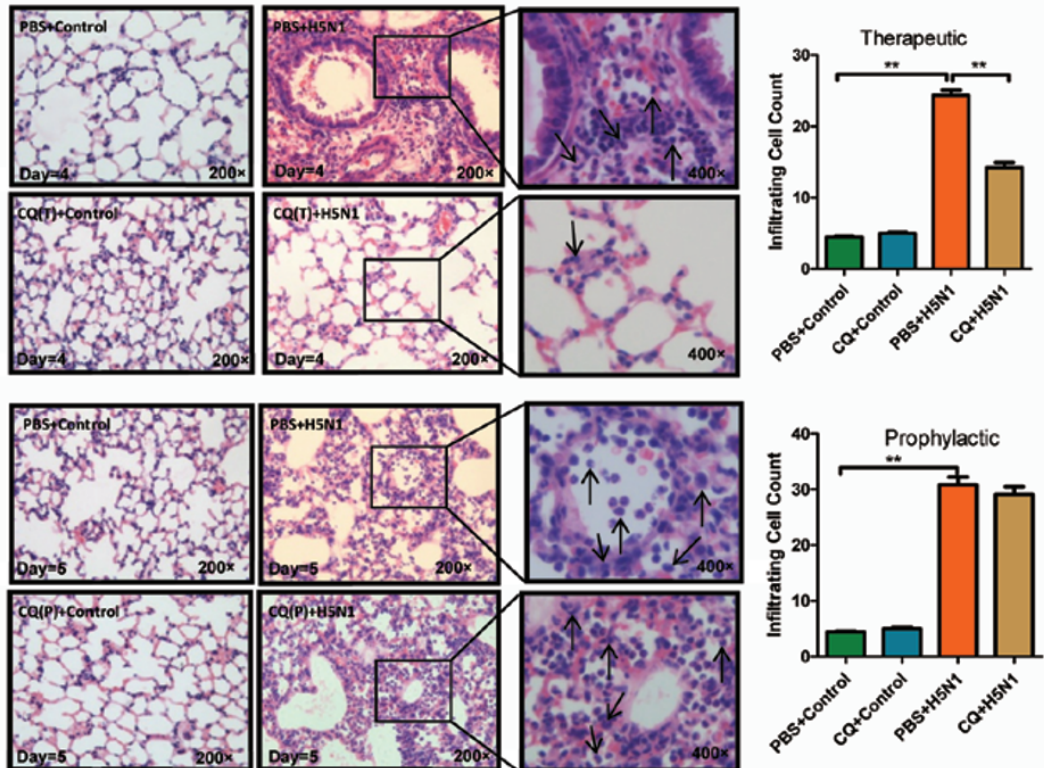

$\mathbf{E}$

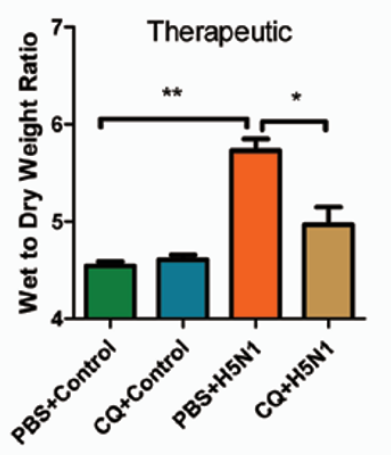

G

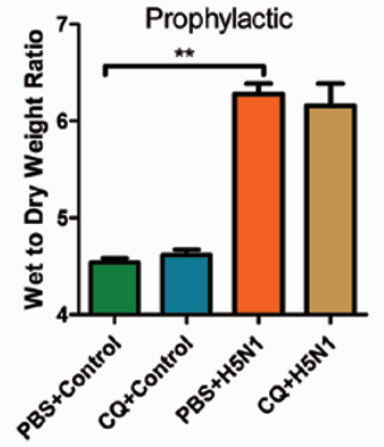

‥ PBS+H5N1

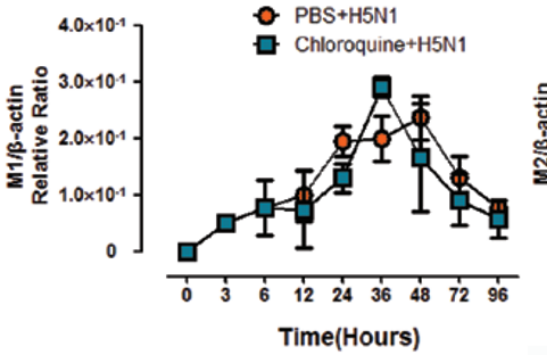

$\mathbf{F}$
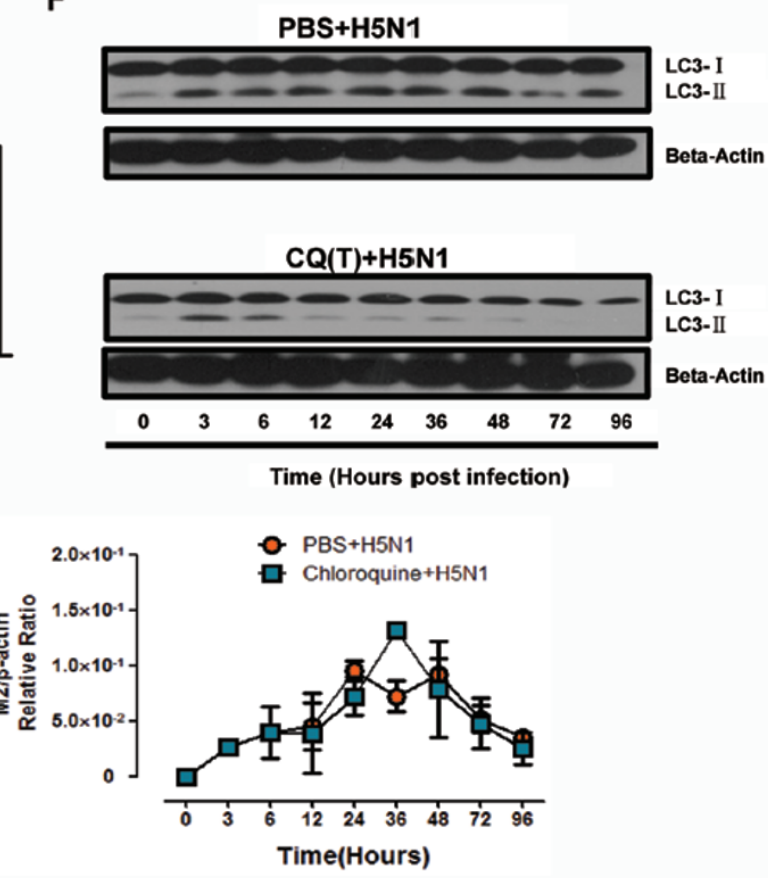
Figure 1 Chloroquine (CQ) is a highly effective therapeutic but not prophylactic agent against avian influenza A H5N1 virus infection in mice. (A) MTS assay of A549 cells treated with 3-MA $(3 \mathrm{mM})$ or chloroquine $(3,10$ or $30 \mu \mathrm{M}) 1 \mathrm{~h}$ before or treated with chloroquine $(30 \mu \mathrm{M})$ 1, 3, $6 \mathrm{~h}$ after infection with control or H5N1 virus (4 MOI) for $48 \mathrm{~h}$. (B) Survival rates of BALB/c mice receiving therapeutic treatment of $C Q$ (i.p. $50 \mathrm{mg} / \mathrm{kg}$ ) or vehicle control for $6 \mathrm{~h}$ and then once per day for 1 week after the intratracheal instillation of vehicle control or $\mathrm{H} 5 \mathrm{~N} 1$ virus $\left(10^{6} \mathrm{TCID}_{50}\right)$ and survival rates for the prophylactic treatment of $\mathrm{CQ}$ (i.p. $\left.50 \mathrm{mg} / \mathrm{kg}\right) 2 \mathrm{~h}$ and $0.5 \mathrm{~h}$ before the intratracheal instillation of vehicle control or $\mathrm{H} 5 \mathrm{~N} 1$ virus $\left(10^{6} \mathrm{TCID}_{50}\right)(n=10$ mice per group). (C) Changes in body weights of BALB/C mice receiving therapeutic treatment of $C Q$ as described above. The values are means \pm SEM from ten mice. (D) Representative lung histopathology of BALB/c mice with therapeutic or prophylactic treatment of CQ as described above. Lung tissues were obtained on day 4 or 5 after virus infection. The black arrows pointed at the monocytes and neutrophils of the infiltrating cells. The bar graph shows the mean number of lung infiltrating cells \pm SEM from 100 microscopic fields of each group (Original magnification was 200x; microscopic fields from the lungs of BALB/c mice infected with $\mathrm{H} 5 \mathrm{~N} 1$ virus with or withour therapeutic or prophylactic treatment of $C Q$ were partially magnified to $400 x ; 100$ fields were analyzed. $n=3$ mice per group). (E) Wet/dry weight ratios of the lungs of BALB/c mice with therapeutic or prophylactic treatment of $C Q$ as described above. Lung tissues were obtained on day 4 or 5 after virus instillation ( $n=4-6$ mice per group). (F) Western blot analysis of LC3-I and LC3-II in mouse lung tissue receiving therapeutic treatment of CQ as described above. Blots were analyzed with antibodies against the indicated proteins. (G) Real-time quantitative PCR analysis of M1's and M2's relative ratios to $\beta$-actin in mouse lung tissue receiving therapeutic treatment of CQ as described above ( $n=3$ mice per group).

$\mathrm{CQ}$ acts to raise the lysosomal $\mathrm{pH}$, leading to the inhibition of both the fusion of autophagosomes with lysosomes and lysosomal protein degradation [7]. As a lysosomotropic agent, CQ could prevent endosomal acidification, which might inhibit influenza virus endocytotic cell entry $[10,11]$. CQ was therefore proposed to be a candidate prophylactic agent for influenza virus. However, government-sponsored clinical trials have shown insufficient prophylactic effects against influenza infection [12], which is consistent with our mouse results. CQ could also inhibit the innate immune responses through TLR signaling pathways and act as novel antagonists to chemokine receptor CXCR4 in pancreatic cancer $[9,13]$. We have shown that CQ treatment clearly inhibited the autophagy in mouse lung induced by avian influenza A H5N1 virus while the virus loads and proinflammatory cytokines were not significantly affected (Figure 1F, 1G, Supplementary information, Figure S1). Further studies are necessary to elucidate the precise molecular mechanisms by which CQ ameliorates murine acute lung injury induced by avian influenza A H5N1 virus.

This notwithstanding, our study strongly suggests that CQ (and potentially its derivatives) should be evaluated as a candidate drug for clinical treatment of H5N1infected patients. Moreover, a systematic screening of common clinical drugs for potential autophagy inhibitors may lead to the identification of other novel treatments against avian flu.

\section{Acknowledgments}

This work is supported by the Ministry of Science and Technology of China (2009CB522105), the National Natural Science Foundation of China (81230002), and the 111 Project (B08007). $\mathrm{CJ}$ is a Hsien Wu professor of Biochemistry.

\section{Yiwu Yan ${ }^{*}, 1$, Zhen Zou ${ }^{*}, 1$, Yang Sun ${ }^{1}$, Xiao $\mathrm{Li}^{2}$, Kai-Feng $\mathrm{Xu}^{3,4}$, Yuquan $\mathrm{Wei}^{5}$, Ningyi $\mathrm{Jin}^{2}$, Chengyu Jiang ${ }^{1,4}$}

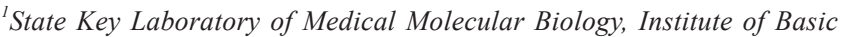 \\ Medical Sciences, Chinese Academy of Medical Sciences, Department of \\ Biochemistry and Molecular Biology, Peking Union Medical College, Tsin- \\ ghua University, Beijing 100005, China ${ }^{2}$ Genetic Engineering Laboratory, \\ Institute of Military Veterinary, Academy of Military Medical Sciences, \\ Changchun, Jilin 130062, China; ${ }^{3}$ Peking Union Medical College Hospi- \\ tal, No.1 Shuaifuyuan, Beijing 100730, China; ${ }^{4}$ Center for Translational \\ Medicine, Peking Union Medical College Hospital, No.1 Shuaifuyuan, \\ Beijing 100730, China; ${ }^{5}$ State Key Laboratory, Biotherapy and Cancer \\ Center, West China Hospital, Sichuan University, Chengdu, Sichuan \\ 610041, China \\ *These two authors contributed equally to this work. \\ Correspondence: Chengyu Jiang ${ }^{\mathrm{a}}$, Ningyi Jin ${ }^{\mathrm{b}}$ \\ ${ }^{a}$ Tel: 86-10-69156908; Fax: 86-10-65276551 \\ E-mail: jiang@pumc.edu.cn \\ ${ }^{b}$ E-mail: ningyij@yahoo.com
}

\section{References}

1 Herfst S, Schrauwen EJ, Linster M, et al. Science 2012; 336:15341541.

2 Imai M, Watanabe T, Hatta M, et al. Nature 2012; 486:420-428.

3 Enserink M. Science 2011; 334:1192-1193.

4 Bauer TT, Ewig S, Rodloff AC, et al. Clin Infect Dis 2006; 43:748756.

5 Wang H, Jiang C. Sci China C Life Sci 2009; 52:459-463.

6 Sun Y, Li C, Shu Y, et al. Sci Signal 2012; 5:ra16.

7 Carew JS, Espitia CM, Esquivel JA 2nd, et al. J Biol Chem 2011; 286:6602-6613.

8 Solomon VR, Lee H. Eur J Pharmacol 2009; 625:220-233.

9 Kim J, Yip ML, Shen X, et al. PLoS One 2012; 7:e31004.

10 Savarino A. Lancet Infect Dis 2011; 11:653-654.

11 Wang H, Jiang C. Sci China C Life Sci 2009; 52:464-469.

12 Paton NI, Lee L, Xu Y, et al. Lancet Infect Dis 2011; 11:677-683.

13 Lund J, Sato A, Akira S, et al. J Exp Med 2003; 198:513-520.

(Supplementary information is linked to the online version of the paper on the Cell Research website.) 